\title{
Influence of Pre-Plasma on Accelerated Protons in Ultraintense Laser Interaction with Not-So-Thin Foils
}

Leonida Gizzi ( $\nabla$ leonidaantonio.gizzi@cnr.it)

Consiglio Nazionale delle Ricerche

\section{Elisabetta Boella}

Lancaster University

\section{Luca Labate}

Consiglio Nazionale delle Ricerche

\section{Federica Baffigi}

Intense Laser Irradiation Laboratory

\section{Pablo Bilbao}

Lancaster University, Bailrigg

\section{Fernando Brandi}

Intense Laser Irradiation Laboratory

Gabriele Cristoforetti

Intense Laser Irradiation Laboratory

Alberto Fazzi

Politecnico di Milano https://orcid.org/0000-0001-5384-9962

Lorenzo Fulgentini

Intense Laser Irradiation Laboratory

Dario Giove

INFN-LASA, Segrate

Petra Koester

Intense Laser Irradiation Laboratory

\section{Daniele Palla}

Intense Laser Irradiation Laboratory

Paolo Tomassini

Intense Laser Irradiation Laboratory

\section{Article}

Keywords: Pre-plasma, Protons, Foils, Laser interaction

Posted Date: September 18th, 2020 
DOI: https://doi.org/10.21203/rs.3.rs-68040/v1

License: (a) (i) This work is licensed under a Creative Commons Attribution 4.0 International License. Read Full License 


\title{
Influence of pre-plasma on accelerated protons in ultraintense laser interaction with not-so-thin foils
}

\author{
Leonida A. Gizzi ${ }^{1,2, @ ~}$, Elisabetta Boella ${ }^{3,4,8}$, Luca Labate ${ }^{1,2, *}$, Federica Baffigi ${ }^{1}$, Pablo J. \\ Bilbao $^{3}$, Fernando Brandi ${ }^{1}$, Gabriele Cristoforetti ${ }^{1}$, Alberto Fazzi ${ }^{5,6}$, Lorenzo Fulgentini ${ }^{1}$, \\ Dario Giove ${ }^{7}$, Petra Koester ${ }^{1}$, Daniele Palla ${ }^{1}$, and Paolo Tomassini ${ }^{1}$
}

\author{
${ }^{1}$ Intense Laser Irradiation Laboratory, INO-CNR, Pisa, Italy \\ ${ }^{2}$ INFN, Sez. Pisa, Italy \\ ${ }^{3}$ Physics Department, Lancaster University, Bailrigg, Lancaster LA1 4YW, UK \\ ${ }^{4}$ Cockcroft Institute, Sci-Tech Daresbury, Keckwick Lane, Warrington WA4 4AD, UK \\ ${ }^{5}$ Dipartimento di Energia, Politecnico di Milano, Italy \\ ${ }^{6}$ INFN, Sezione di Milano, Italy \\ ${ }^{7}$ INFN-LASA, Segrate, Italy \\ @leonidaantonio.gizzi@cnr.it \\ 1e.boella@lancaster.ac.uk \\ *luca.labate@ino.cnr.it
}

\begin{abstract}
We report on recent experimental results on proton acceleration from laser interaction with foil targets at ultra-relativistic intensities. We show a three-fold increase in the proton cut-off energy when a micrometer scale-length pre-plasma is introduced by irradiation with a low energy femtosecond pre-pulse. The foil target is sufficiently thick to prevent disruption of the sheath field at the rear surface by the shock launched by the pre-pulse. Measurements are compared with accurate, numerical hydrodynamic and Particle-In-Cell simulations where the role of the finite plasma scale-length at the laser-target interface is taken into account and the role of stochastic heating in enhancing fast electron production is discussed.
\end{abstract}

\section{Introduction}

Ion acceleration driven by ultraintense lasers using Target Normal Sheath Acceleration (TNSA) ${ }^{1}$ is establishing itself as a powerful technique to access relatively high energy ion beams in a compact and affordable layout. Since the original investigations, effort has been dedicated ${ }^{2,3}$ to enhance the cut-off energy and the flux of the accelerated ions via a number of advances of laser and target specifications using plasma mirror for ultra-high laser contrast with ultra-thin targets ${ }^{4}$, cryogenic $^{5}$ or nano-structured targets ${ }^{6}$. To date, however, practical exploitation of laser-driven ion acceleration relies heavily on the original TNSA configuration based on thin foil targets and optimized contrast without plasma mirror, possibly operating at the repetition rate required for applications like radiobiology 7,8 , where high dose irradiation is needed for meaningful studies. In this context, great attention is being dedicated to the control of accelerated ions, including energy cut-off, beam divergence, charge and emittance. Target optimisation and engineering, looking at different properties of surface, geometry and conductivity, are becoming crucial in this effort to ensure reliable and reproducible performance of beamlines based on laser-driven acceleration.

From a more fundamental viewpoint, recent investigations to further improve ion acceleration performance focus on the interaction parameters affecting the energy density of laser-heated fast electrons, namely their divergence, peak current and temperature. It is well known that fast electron generation is strongly modified by the size of the laser focal spot ${ }^{9,10}$, and more effectively by the scale-length of the plasma at the vacuum-target interface. Indeed, several modelling studies based on Particle-In-Cell (PIC) simulations predict a significant increase of the proton cut-off energy with optimized pre-plasma conditions. In Sentoku et al. ${ }^{11}$, simulations were used to investigate fast electron heating in the standing wave occurring in the pre-plasma from superposition of incident and reflected wave, showing a linear increase of the ion energy cut-off with the scale-length of the pre-plasma. Similar results were shown in a more recent modelling study ${ }^{12}$ where the role of temporal pulse shape was also explored. The role of standing waves was then further investigated in a colliding pulses configuration with two pulses incident on the target from opposite incident angles ${ }^{13}$, showing enhancement in the fast electron temperature and ion energy cut-off for both ultra-high contrast laser systems using double plasma mirror. In the same study, a strong linear increase of the cut-off energy with the scale-length of the pre-plasma is shown for both colliding pulse and single pulse at constant energy, with the highest proton cut-off energy occurring for a micrometer scale-length plasma. More in details, in Sentoku 
et al. ${ }^{11}$, a moderate increase of the proton energy up to 1.5 times was found at mildly relativistic intensities of $10^{18} \mathrm{~W} / \mathrm{cm}^{2}$ at $1.053 \mu \mathrm{m}$ wavelength for a micrometer-scale pre-plasma. The increase was explained as due to the stochastic heating of electrons in the standing wave structure responsible for an enhanced ponderomotive acceleration of electrons. In the same study it is shown that this enhancement of proton energy can be expected to grow to a factor of 4 or more for longer scale-length and eventually saturate above 6-8 $\mu \mathrm{m}$ scale-length. On the other hand, this enhancement factor may be partially reduced at ultra-relativistic intensities due to the $\propto \sqrt{I}$ scaling compared to the $\propto I$ of moderately or sub-relativistic intensities. In Ferri et al. ${ }^{13}$ simulations at a higher intensity of $7 \times 10^{19} \mathrm{~W} / \mathrm{cm}^{2}$ at $0.8 \mu \mathrm{m}$ wavelength $\left(\mathrm{a}_{0}=5.7\right)$ leads approximately to a three-fold increase of the proton cut-off for single pulse irradiation of a $3 \mu \mathrm{m}$ thick Al target and for a density scale-length of $0.6 \mu \mathrm{m}$. In Kumar et al. ${ }^{12}$ simulations at ultra-relativistic intensities of $10^{20} \mathrm{~W} / \mathrm{cm}^{2}$ and $\mathrm{a}_{0}=10.6$, very similar to the value of our experiment, were investigated. In this intensity regime PIC simulations show that in the presence of a pre-plasma consisting of a $10 \mu \mathrm{m}$ linear density ramp, the proton cut-off energy is expected to be approximately $8.5 \mathrm{MeV}$, while without the pre-plasma, the cut-off energy is $5 \mathrm{MeV}$, showing again an enhancement of approximately a factor 1.7.

In spite of the detailed theoretical predictions, to date, a clear experimental evidence of such enhancement is still lacking. As clarified in the discussion below, this is mainly due to the complex interplay occurring in real laser-target interactions, between plasma formation on the front side, typically driven by ad-hoc pre-pulse laser irradiation, and the deformation/disruption of the target rear side where ion acceleration mainly occurs.

Here we present new results in which the activation of a micrometer scale-length pre-plasma leads to a three-fold increase of the cut-off energy of accelerated protons compared to measurements obtained without pre-plasma, using optimized laser contrast without plasma mirror ${ }^{14}$. As discussed below, the use of a very low energy $(\mathrm{mJ})$ femtosecond pre-pulse to generate the pre-plasma, combined with a relatively thick foil target, ensures controllable pre-plasma formation at the target front side with no shock perturbation of the target rear side, so that the results can be directly compared with the case of no pre-plasma, without uncontrolled disruption of the sheath field due to shock heating. The results are modelled using PIC simulations that account for the presence of a finite scale-length pre-plasma at the vacuum-target interface. The properties of the pre-plasma are also investigated using hydrodynamic modelling. Consistently with previous studies, our numerical simulations point at the presence of stochastic heating of fast electrons and offer useful insight on the potential impact of key interaction parameters for further optimization and control of efficient and robust TNSA ion sources.

\section{Results and discussion}

Fig. 1 shows two sets of proton spectra obtained with the Thomson Parabola Spectrometer (TPS) (see Methods) on $10 \mu \mathrm{m}$ Al targets and $25 \mu \mathrm{m}$ Ti targets, respectively. The three spectra with $10 \mu \mathrm{m}$ Al targets were obtained without the pre-plasma and with the standard contrast of our laser ${ }^{14}$ and show a cut-off energy around $6 \mathrm{MeV}$. In contrast, the four spectra with $25 \mu \mathrm{m}$ Ti targets were obtained with the pre-plasma generated (see Methods) by a short pre-pulse arriving 10.4 ns before the main pulse. According to hydrodynamic simulations (see Methods), at the time on main pulse interaction, the scale-length of the pre-plasma is $7 \mu \mathrm{m}$ at the classical critical density $\left(1.7 \times 10^{21} \mathrm{~cm}^{-3}\right)$ for the $800 \mathrm{~nm}$ laser light and $4.5 \mu \mathrm{m}$ at the relativistic critical density $\left(1.8 \times 10^{22} \mathrm{~cm}^{-3}\right)$.

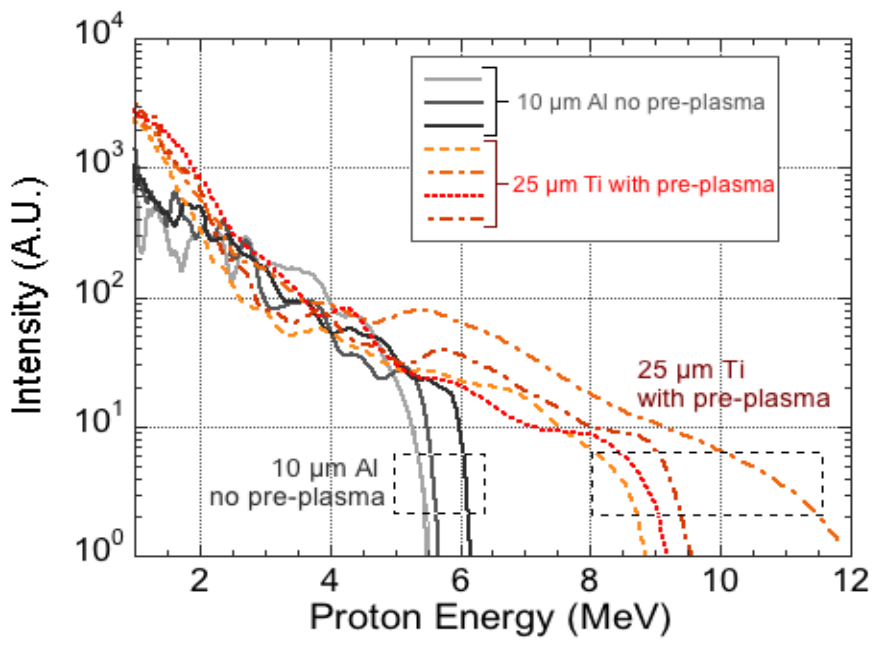

Figure 1. Comparison of experimental proton spectra obtained from the TPS for $10 \mu \mathrm{m}$ thick Al targets without pre-plasma and $25 \mu \mathrm{m}$ thick Ti target with the pre-plasma. 
With this pre-plasma the $25 \mu \mathrm{m}$ Ti spectra of Fig.1 show a cut-off energy in the range between $\sim 9$ and $12 \mathrm{MeV}$, namely up to a 2 -fold increase with respect to the $10 \mu \mathrm{m} \mathrm{Al}$ target. These results clearly show that, in spite of the significant increase of target thickness compared with the $10 \mu \mathrm{m} \mathrm{Al}$, the presence of the pre-plasma modifies significantly the proton spectrum obtained with the $25 \mu \mathrm{m} \mathrm{Ti}$, with a strong enhancement of the maximum energy of accelerated protons. A systematic increase of the signal at low energy is also visible for the Ti data compared with Al targets. The $25 \mu \mathrm{m}$ Ti plots of Fig. 1 also demonstrate that the observed increase of the cut-off energy in the presence of a pre-plasma is repeatable and statistically significant.

In order to evaluate a possible contribution from the different target materials, shots were taken with Ti targets of different thickness. Fig. 2 shows the proton spectra obtained from the Time Of Flight (TOF) signals taken with the SiC detector (see Methods) from the irradiation of Ti targets with thickness ranging from 2 to $25 \mu \mathrm{m}$. All the data were taken without the pre-plasma, except the purple line with cut-off at $10 \mathrm{MeV}$ that was obtained with the pre-plasma. According to these plots, the cut-off energy without the pre-plasma is maximum around $7 \mathrm{MeV}$ for a $5 \mu \mathrm{m}$ Ti target and drops slightly to $6 \mathrm{MeV}$ for 12.5 and to $4 \mathrm{MeV}$ for $25 \mu \mathrm{m}$ Ti.

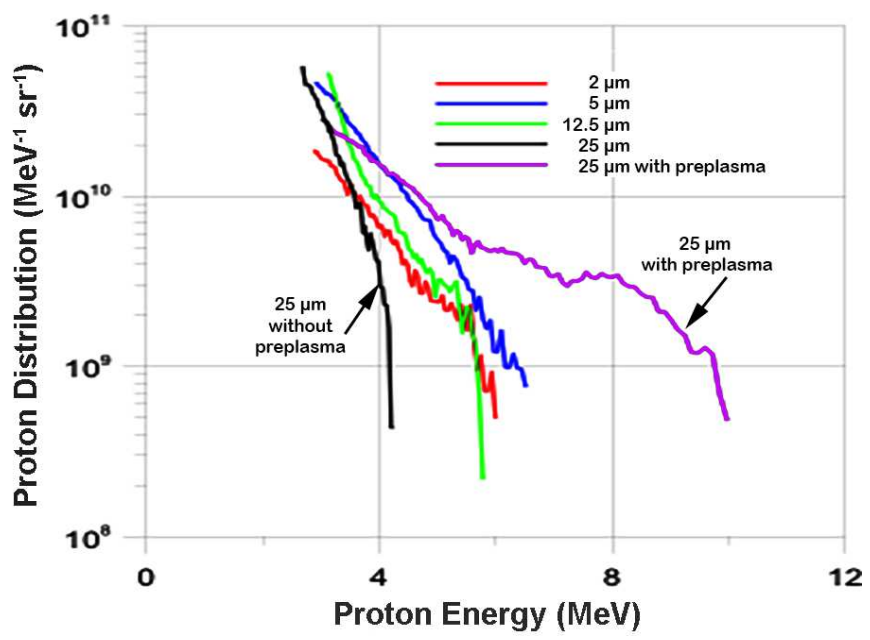

Figure 2. Comparison of experimental spectra obtained from deconvolution of the temporal evolution of the TOF signals from a set of Titanium foil targets of different thickness of 2, 5, 12.5 and $25 \mu \mathrm{m}$. All data were taken without the pre-plasma, except the purple line for $25 \mu \mathrm{m}$ with cut-off energy at $10 \mathrm{MeV}$.

This is consistent with the expected behaviour for TNSA accelerated protons where cut-off energy is expected to decrease with increasing target thickness ${ }^{2,3}$. As already observed in our experimental conditions ${ }^{14}$, the cut-off energy also decreases for the thinnest target of $2 \mu \mathrm{m}$ Ti. This is expected due to the finite temporal contrast of our laser system at the ps level as previously discussed in details ${ }^{14}$. Similar cut-off energies were obtained for $2.4 \mu$ mAluminium targets that showed a cut-off energy of $<4 \mathrm{MeV}^{14}$.

According to Fig.2, in contrast with the observed reduction with increasing thickness, the cut-off energy of the $25 \mu \mathrm{m}$ Ti target in the presence of the pre-plasma exhibits the highest value of $10 \mathrm{MeV}$. Notably, this value is approximately 2.5 times the value obtained for the same target thickness without the pre-plasma. These results confirm those obtained with the TPS, as shown in Fig.1 where a cut-off energy as high as $12 \mathrm{MeV}$ is shown, corresponding to a three-fold increase of the cut-off energy without the pre-pulse for the same $25 \mu \mathrm{m}$ Ti target thickness.

We observe that the highest cut-off energy with the pre-plasma was found only for the $25 \mu \mathrm{m}$ thick Titanium target, namely for a significantly thicker target, while in all other explored cases of thinner targets, a lower or similar cut-off energy was found in the pre-plasma case, compared with the no pre-plasma case, when the same target thickness was used. As discussed below, this is due to the effect of the shock launched by the pre-pulse used to generate the pre-plasma that, for thinner targets, reaches the rear surface before the arrival of the main pulse, disrupting the sheath field.

As anticipated, the role of a pre-plasma in the acceleration of protons was highlighted in previous simulation studies to identify possible mechanisms of enhancement of fast electron heating. For example, a comparison with Kumar et al., ${ }^{12}$ shows that the measured values of the cut-off energy for the two cases with and w/o the pre-plasma are in a qualitative agreement with the calculated ones, although the enhancement factor $(3 \mathrm{x})$ observed in our study is significantly higher than the one $(1.7 x)$ found $i^{12}$. Assuming that the enhancement factor is entirely due to the presence of the pre-plasma, the reason for this discrepancy should be explored in the interaction with the pre-plasma. We observe that the scale-length of $10 \mu \mathrm{m}$ used in those 

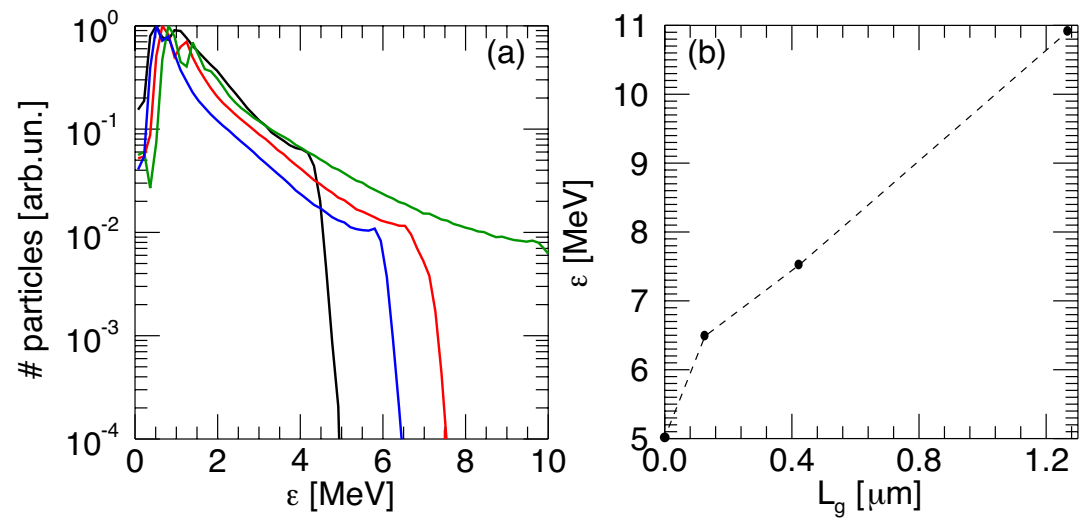

Figure 3. 3D PIC simulation results: (a) Proton energy spectra for a $10 \mu \mathrm{m} \mathrm{Al}$ target for different pre-plasma scalength: $L_{g}=0.13$ (blue), 0.42 (red) and $1.27 \mu \mathrm{m}$ (green). The black curve corresponds to a sharp plasma-vacuum transition. (b) Cut-off energy vs pre-plasma scalength for the cases in (a).

simulations is already in the saturation regime found in Sentoku et al., ${ }^{11}$ and therefore, we can reasonably assume that this discrepancy should not be attributed to a change in the scale-length. On the other hand, in the same paper ${ }^{12}$ the role of laser pulse longitudinal asymmetry is discussed and, a pulse with a faster leading edge is found to yield a cut-off energy as high as 12 $\mathrm{MeV}$, a value that would be in an excellent agreement with the one measured in our experiment. Indeed, laser pulse longitudinal asymmetry can arise from propagation of intense laser pulses in underdense plasmas, due to non-linear changes of the refractive index. It has been shown ${ }^{15}$ that self-steepening at the front of the laser pulse can occur already at mildly relativistic intensities with a skewness rate that increases with laser intensity up to the complete blowout regime.

Motivated by these considerations we carried out 2D and 3D PIC simulations (see Methods) to establish a reference modelling for our ion acceleration experiment with the optimized laser contrast (i.e. no mJ pre-pulse) and to identify the role of the pre-plasma on the observed ion spectra, with a pre-plasma scale-length ranging from approximately 0.13 up to $5 \mu \mathrm{m}$, to account for the expected scale-length at the relativistic critical density predicted by our hydrodynamic simulations in the presence of the pre-pulse (see Methods). The spectrum of accelerated protons obtained from 3D PIC simulations for a $10 \mu \mathrm{m} \mathrm{Al}$ for the case of no pre-plasma and for values of the plasma scale-length of $L_{g}=0.13 \mu \mathrm{m}, L_{g}=0.42 \mu \mathrm{m}$ and $L_{g}=1.27 \mu \mathrm{m}$ are shown in Fig.3(a), while Fig.3(b) reports the proton cut-off energy for the same cases. According to these results, simulations predict a cut-off energy just below $5 \mathrm{MeV}$ for the pre-plasma free case. This value increases up to $7.5 \mathrm{MeV}$ for the $420 \mathrm{~nm}$ plasma scale-length and exceeds $10 \mathrm{MeV}$ for the $1.27 \mu \mathrm{m}$ scale-length. These simulations show a linear dependence of the cut-off energy with the pre-plasma scale-length, predicting a cut-off energy increasing rapidly with the pre-plasma scale-length, with a two-fold increase for a scale-length of $1.27 \mu \mathrm{m}$. A comparison with our experimental results of Fig. 1 for the case of 10 $\mu \mathrm{m} \mathrm{Al}$ with optimized laser contrast (no pre-plasma), yielding a measured cut-off energy of $6.0 \mathrm{MeV}$, shows a similar value for a pre-plasma scale-length of approximately $100 \mathrm{~nm}$. As anticipated, this scale-length is consistent with the presence of the ps pedestal of our laser pulse. In fact, taking into account the ps contrast our laser system ${ }^{14}$, we expect the formation of a short scale-length pre-plasma a few ps before the laser peak. Considering a pre-plasma expanding at the ion-acoustic wave speed, we estimate a density scale-length at the critical density of the order of $100 \mathrm{~nm}$, a value that is fully consistent with the scale-length expected from the numerical simulations results discussed above.

Fig. 3 confirms that in the case of a $10 \mu \mathrm{m} \mathrm{Al}$ target the proton cut-off energy could exceed $10 \mathrm{MeV}$ already for a modest pre-plasma scale-length just above $1.27 \mu \mathrm{m}$. However, we stress here that in these and other similar PIC simulations, the presence of the pre-plasma in front of the target is included ad hoc and self-consistency of the results should be discussed. In reality, the formation of any pre-plasma generated on the target surface by either the ps pedestal or by a custom pre-pulse, also involves the bulk of the target. The shock generated at the target surface by any pre-pulse interaction eventually reaches the rear surface of the target, disrupting the formation of the TNSA field. This problem can only be neglected in the case of sufficiently thick targets, where shock break-out at the target rear face occurs late, so that TNSA and ion acceleration take place before any disruption of the target rear surface has occurred.

The role of shock propagation in the bulk target induced by pre-pulse irradiation was investigated with our hydrodynamic simulations (see Methods). We found a shock wave propagation speed of $1.81 \mu \mathrm{m} / \mathrm{ns}$ for the $\mathrm{Al}$ target. Consequently, in the case of the $10 \mu \mathrm{m} \mathrm{Al}$ target, shock break-out occurs $5.5 \mathrm{~ns}$ after pre-pulse irradiation, that is before main pulse irradiation that in our experiment occurs at $10.4 \mathrm{~ns}$. Therefore, in our experimental conditions, the enhancement seen in PIC simulations 

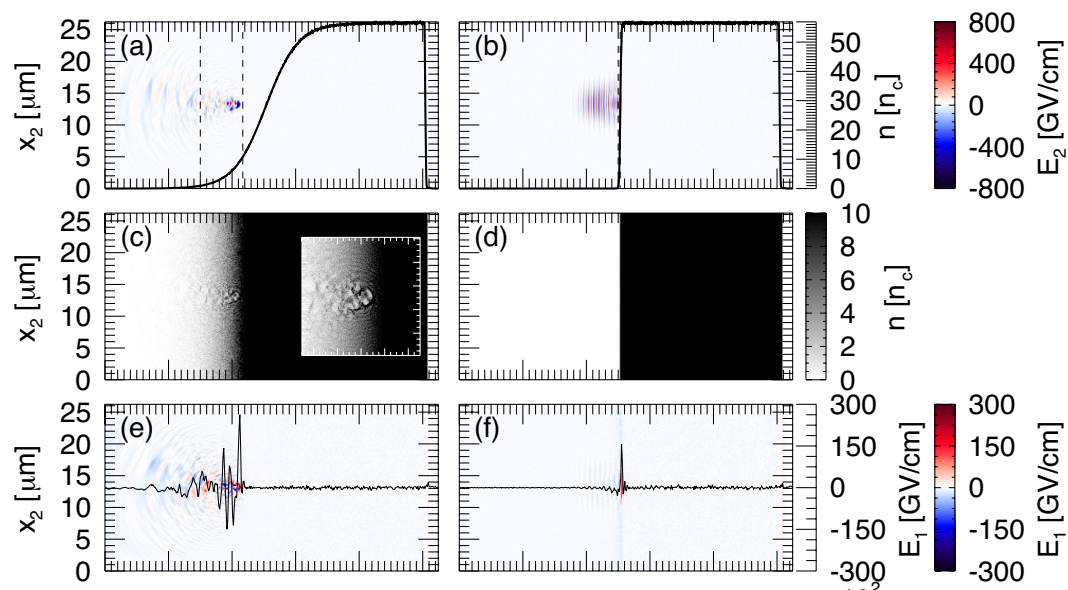

\author{
10
}

$\stackrel{\widetilde{c}}{\check{c}}$
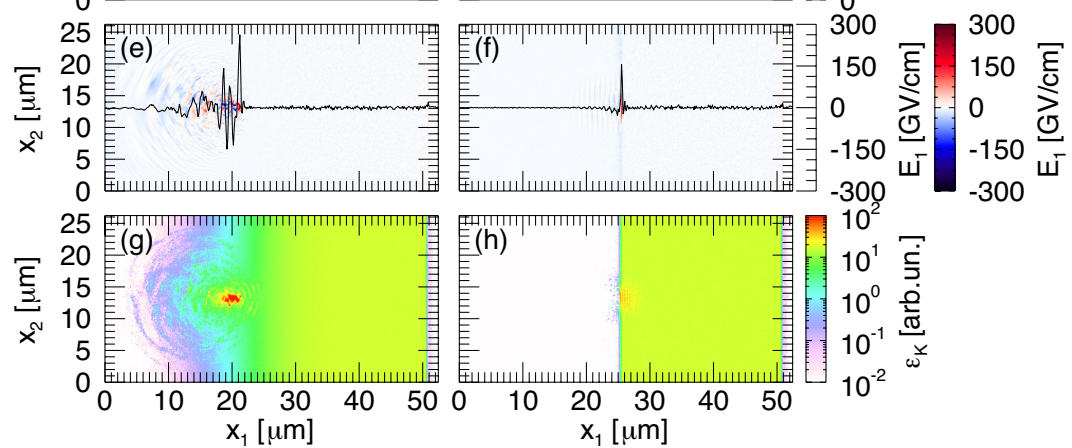

Figure 4. 2D PIC simulations: (a, b) Laser electric field and longitudinal density profile (black solid line), (c, d) electron density, (e, f) longitudinal electric field and $\left(\mathrm{g}, \mathrm{h}\right.$ ) electron kinetic energy density at $t=0.11 \mathrm{ps}$ for a Ti plasma with $L_{g}=5$ (left column) and $0.13 \mu \mathrm{m}$ (right column). The black dashed lines in (a, b) denote the position of the critical density and the relativistic critical density. The inset in (c) is an enlargement showing details of the electron cavities on the target surface. The black solid lines in $(e, f)$ represent the electric field lineout in the middle of the simulation box.

for the $10 \mu \mathrm{m} \mathrm{Al}$ target with $1.27 \mu \mathrm{m}$ scale-length could not be observed because disruption of the rear surface would occur before main pulse irradiation. In contrast, for Ti targets the hydrodynamic code predicts a shock propagation speed of 1.66 $\mu \mathrm{m} / \mathrm{ns}$ that, for the $25 \mu \mathrm{m}$ Ti target, gives a shock break-out time at the rear surface of $15 \mathrm{~ns}$ after pre-pulse irradiation. In our experimental conditions this corresponds to $4.6 \mathrm{~ns}$ after the main pulse irradiation, thus allowing TNSA to take place without perturbation. These considerations indeed show that the presence of the pre-plasma for Al targets below $19 \mu \mathrm{m}$ and Ti targets below approximately $17.5 \mu \mathrm{m}$ are subject to the effect of the shock break-out at the rear face and disruption of the TNSA field. This also explains why higher cut-off energy was only found for the $25 \mu \mathrm{m}$ thickness Ti among all explored targets. Incidentally we observe that the TNSA proton cut-off energy for sharp vacuum-target interface is indeed expected to decrease with target thickness, thus making such relatively thick targets, in general, not ideal for efficient proton/ion acceleration. On the other hand, both simulations and experimental data clearly show that for a sufficiently large scale-length pre-plasma, the gain in cut-off energy due to interaction with the pre-plasma can largely overcome such losses and lead to record cut-off values.

In order to investigate the interaction with a more extended pre-plasma, we also carried out 2D PIC simulations (see Methods) with a scale-length beyond the value of $1.27 \mu \mathrm{m}$ explored above with 3D simulations. Being computationally much more affordable that the 3D simulations, 2D simulations allowed us to explore the role of longer pre-plasma gradient in front of the target with scale-lengths of the order of those predicted by hydrodynamic simulations (see Methods). In Fig.4 the results from a simulation with a long pre-plasma $\left(L_{g}=5 \mu \mathrm{m}\right)$ are compared with results obtained with $L_{g}=0.13 \mu \mathrm{m}$. When a long plasma gradient is present in front of the target, the laser penetrates through the underdense plasma (Fig.4(a)), where it undergoes self-focusing and steepening. When the laser reaches the plasma region where $n_{c} \leq n \leq a_{0} n_{c}$ with $n$ electron density and $a_{0} n_{c}$ relativistic critical density, it expels electrons forming semi-circular cavities (Fig.4(c) $)^{16,17}$. It is interesting to notice that nothing of this is observed when the laser reaches the target surface when $L_{g}=0.13 \mu \mathrm{m}$, corresponding to a nearly sharp, almost unperturbed, vacuum-plasma transition (Fig.4(b)). In the latter case the ponderomotive force associated with the laser is not large enough to push the target surface inward and bore a hole (Fig.4(d)) and the laser is reflected back by the opaque target.

The complex laser dynamics in the plasma section across the critical density for the case $L_{g}=5 \mu \mathrm{m}$ leads to the formation of a standing wave. The latter is due to a superposition between the incident and reflected electromagnetic wave ${ }^{11,12}$. Here the role of the angle of incidence $\left(15^{\circ}\right.$ in our case) has been discussed recently ${ }^{18}$ showing effective contribution of stochastic heating for relatively long scale-length and even larger angles of incidence. Signatures of the standing wave are clearly visible in Fig.4(e) while such signatures are absent in Fig.4(f), where the longitudinal electric field is plotted for $L_{g}=5$ and $0.13 \mu \mathrm{m}$, 

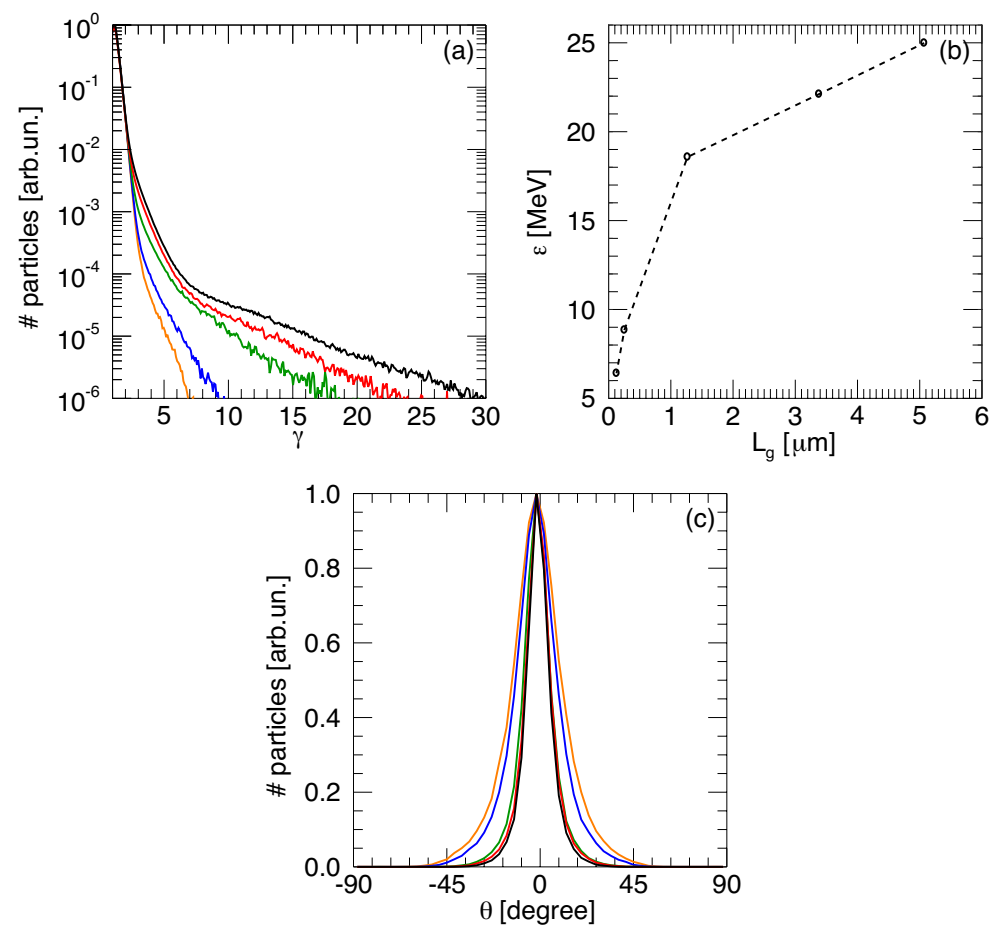

Figure 5. 2D PIC simulations results: (a) Electron distribution for density scale-length $L_{g}=0.13$ (orange), 0.25 (blue), 1.27 (green), 3.38 (red) and $5.07 \mu \mathrm{m}$ (black). (b) Proton cut-off energy vs pre-plasma scale length and (c) proton angular distribution for the cases in (a).

respectively. Electrons undergo stochastic motion in the standing wave ${ }^{11,19}$ resulting in an overall more efficient electron heating, with a larger number of electrons reaching higher energies (Figs.4(g) and (h)). The improved coupling between the laser pulse and the electrons in the presence of an extended pre-plasma is clearly visible in Fig.5(a), which shows the electron distribution in the whole box after electrons have recirculated through the target, for different values of $L_{g}$. All the spectra are made up by a cold and a hot electron component. While the cold electrons have similar temperatures, the hot electrons exhibit higher temperatures for longer plasma gradients, with temperatures varying between $0.31 \mathrm{MeV}$ for $L_{g}=0.13 \mu \mathrm{m}$ and $1.49 \mathrm{MeV}$ for $L_{g}=5.07 \mu \mathrm{m}$. As a consequence, in the latter cases, the sheath field is enhanced and protons attain higher energies (Fig.5(b)). As noted in Fig.3, the proton cut-off energy increases linearly with $L_{g}$. However, here we observe that for $L_{g} \geq 1.27 \mu \mathrm{m}$, the maximum proton energy increases at a slower rate compared to shorter pre-plasma gradients. A similar behaviour was reported also in Sentoku et al. ${ }^{11}$, but for higher values of $L_{g}$. On one side this difference might be due to the use of a lower laser intensity in their case. On the other side it is an indication that the plasma scale-length used in our experimental conditions may already be in the saturation regime.

A comparison with the 3D simulations of Fig. 3 shows that for the same scale-length of $1.27 \mu \mathrm{m}, 2 \mathrm{D}$ simulations predict a higher cut-off energy of approximately $17 \mathrm{MeV}$, instead of $11 \mathrm{MeV}$. This is expected due to the slower decay ${ }^{20}$ of the sheath field in 2D compared to 3D simulations, as also discussed below (see Methods). Therefore a quantitative comparison with experimental values is not straightforward. However, it is clear from these 2D simulation results that the presence of an extended pre-plasma enables a more effective energy coupling to fast electrons that is at the origin of the higher proton cut-off energy recorded for the $25 \mu \mathrm{m}$ Ti target, with $L_{g} \geq 5 \mu \mathrm{m}$ and yielding the observed three-fold increase of the maximum proton energy. Finally, simulations seem to indicate that beside an increase of the cut-off energy, the presence of a long plasma gradient leads to a more collimated proton beam (Fig.5(c)). This could not be verified in our experiment and will be the subject of future investigations.

\section{Methods}

\section{Experimental set up}

The experiment was carried out at the Intense Laser Irradiation Laboratory using the ILIL-PW Ti:Sa laser installation ${ }^{21}$ based on Chirped Pulse Amplification (CPA) ${ }^{22}$. The laser pulse energy was up to $4.3 \mathrm{~J}$ on target with $27 \mathrm{fs}$ pulse duration, with $\lambda=$ 
$0.795 \mu \mathrm{m}$ central laser wavelength. The $100 \mathrm{~mm}$ diameter beam was focused by an F/4.5 Off-Axis Parabolic (OAP) mirror with an angle of incidence of $15^{\circ}$. The focal spot was elliptical, with an average diameter of $4.4 \mu \mathrm{m}$ (FWHM). The nominal intensity on target was up to $2.4 \times 10^{20} \mathrm{~W} / \mathrm{cm}^{2}$ with a relativistic dimensionless parameter $\mathrm{a}_{0}=8.55 \times 10^{-10} \lambda[\mu \mathrm{m}] \mathrm{I}\left[\mathrm{W} / \mathrm{cm}^{2}\right]^{1 / 2}=10.6$ The target consisted of a thin foil mounted on a remotely controlled motorized support with a sub-micrometer positioning precision. Titanium and Aluminium foils were used, with thickness ranging from 2 to $25 \mu \mathrm{m}$. The main laser features and previous ion acceleration measurements are described elsewhere ${ }^{14,21}$, including details of the experimental set up, laser contrast features and ion detectors.

Ion acceleration data were taken using the same set of ion beam diagnostics already presented in ${ }^{14}$, including a TPS and a TOF SiC detector. The $\mathrm{SiC}$ detector was placed at a distance of $136 \mathrm{~cm}$ from the target rear side at $1.4 \mathrm{deg}$ from the target normal and was filtered using a $48 \mu \mathrm{m}$ thick Al foil. The TPS and the TOF detector were used simultaneously so that a cross-comparison of the signals obtained from the two devices was possible ${ }^{23,24}$. We refer to the Supplementary Material for additional information on the comparison between the two detectors.

In the experimental campaign described here we investigated the role of a femtosecond pre-pulse at 10.4 ns before the main pulse, focused with the same OAP, at an intensity relative to the main pulse of $3 \times 10^{-4}$. The pre-pulse was due to the incomplete extinction of the pulse arriving immediately before the main pulse, exiting the regenerative amplifier and then being partially amplified by the amplifier chain of our Chirped Pulse Amplification system. The effect of this pre-pulse on the target was modelled using hydrodynamic simulations to calculate the expected profile of the pre-plasma in the underdense region, at the time of interaction of the main pulse (see below).

\section{Numerical simulations}

Numerical PIC simulations were carried out using the code OSIRIS ${ }^{25}$. In the first set of 3D PIC simulations a $10 \mu \mathrm{m}$ Al foil was considered with a $32 \mathrm{~nm}$ thick $\mathrm{H}^{+}$contaminant layer at the back of the foil. The $\mathrm{Al}$ foil was assumed to be pre-ionised to $\mathrm{Al}^{3+}$ with an initial temperature of $100 \mathrm{keV}$. We verified that different level of target ionization and different initial electron temperature in the range $10-200 \mathrm{KeV}$ did not lead to significant changes on the final $\mathrm{H}^{+}$energy. The density of the $\mathrm{Al}$ layer was 34.14 times the critical density, $\mathrm{n}_{c}=1.76 \times 10^{21} \mathrm{~cm}^{-3}$, while the density of the $\mathrm{H}^{+}$layer on the rear side was 1.14 times the critical density. The front of the target was modelled following a hyperbolic tangent density profile centered at $x=25.3 \mu \mathrm{m}$ with scale-length $L_{g}$ to mimic the presence of the pre-plasma. The laser intensity used for this first set of simulations was $1.6 \times 10^{20} \mathrm{~W} / \mathrm{cm}^{2}$, corresponding to a normalized vector potential $a_{0}=8.6$. The p-polarised pulse was focused on target to a spot size $w_{0}$ of $2.2 \mu \mathrm{m}$ and had a duration at FWHM of $27 \mathrm{fs}$. The simulation box was $200 \times 18 \times 18 \mu \mathrm{m}^{3}$ with a cell size of $60 \times 60 \times 60 \mathrm{~nm}^{3}$, equivalent to approximately 13 points per laser wavelength, with 8 particles per cell. Simulations performed with different resolution and number of particles per cell yielded similar results.

A second set of 2D PIC simulations was performed to carry out a parametric investigation of the role of target thickness and materials. For this set of simulations we employed a laser intensity on target $I=2.4 \times 10^{20} \mathrm{~W} / \mathrm{cm}^{2}\left(\mathrm{a}_{0}=10.6\right)$ and we modelled the interaction of this pulse with a $25 \mu \mathrm{m}$ Ti target having a peak density $n_{T i}=56.9 n_{c}$ and a longitudinal profile similar to that employed in the previous simulation campaign (all the rest of the parameters are kept the same). A simulation box of $607 \times 26 \mu \mathrm{m}^{2}$ with a cell size of $30 \times 30 \mathrm{~nm}^{2}$ equivalent to about 26 points per laser wavelength has been used together with 36 particles per cell. We stress that while it is known that $2 \mathrm{D}$ simulations overestimate the final ion energy ${ }^{20,26}$ and thus make a one-to-one comparison with experimental results not possible, they still offer a valid insight of the underpinning physics $^{26}$.

For the modelling of the pre-pulse generated pre-plasma, we used the $2 \mathrm{D}$ Eulerian hydrocode POLLUX ${ }^{27}$. The code models laser absorption via inverse bremsstrahlung and thermal transport via flux-limited Spitzer-Harm conductivity. Ionization is calculated assuming local thermodynamic equilibrium, while a perfect gas equation of state is used for electrons. According to the simulations, at the time of pre-pulse interaction with the target, the plasma has a temperature of a few eV that rapidly cools down while expanding. We refer to the Supplementary material for additional information on these simulations.

\section{Conclusions}

Our study demonstrates experimentally that a micrometer-size scale-length pre-plasma can strongly enhance proton acceleration driven by ultraintense laser interaction with not-so-thin foil targets, leading to a three-fold increase of the cut-off energy with respect to case with no pre-plasma. For targets sufficiently thick to prevent disruption of the TNSA accelerating field by shock breakout at the rear side, the observed enhancement largely overcomes the reduction of the cut-off energy due to the increased thickness. This behaviour was modelled by detailed PIC simulations and confirmed by our measurements. Our results open the possibility to significantly extend the cut-off energy of robust TNSA based proton sources without plasma mirror or ultra-thin targets and with a minor adjustment of a standard CPA laser front-end. 


\section{References}

1. Snavely, R. A. et al. Intense high-energy proton beams from petawatt-laser irradiation of solids. Phys. Rev. Lett. 85, 2945, DOI: 10.1103/PhysRevLett.85.2945 (2000).

2. Daido, H., Nishiuchi, M. \& Pirozhkov, A. S. Review of laser-driven ion sources and their applications. Reports Prog. Phys. 75, 056401, DOI: 10.1088/0034-4885/75/5/056401 (2012).

3. Macchi, A., Borghesi, M. \& Passoni, M. Ion acceleration by superintense laser-plasma interaction. Rev. Mod. Phys. 85, 751-793, DOI: 10.1103/RevModPhys.85.751 (2013).

4. Kaymak, V. et al. Boosted acceleration of protons by tailored ultra-thin foil targets. Sci. Reports 9, DOI: 10.1038/ s41598-019-55011-2 (2019).

5. Scott, G. G. et al. Dual Ion Species Plasma Expansion from Isotopically Layered Cryogenic Targets. Phys. Rev. Lett. 120, 204801, DOI: 10.1103/PhysRevLett.120.204801 (2018).

6. Ferri, J. et al. Enhancement of laser-driven ion acceleration in non-periodic nanostructured targets. J. Plasma Phys. 86, 1-12, DOI: 10.1017/S0022377819000898 (2019).

7. Bayart, E. et al. Fast dose fractionation using ultra-short laser accelerated proton pulses can increase cancer cell mortality, which relies on functional PARP1 protein. Sci. Rep. 9, 1-10, DOI: 10.1038/s41598-019-46512-1 (2019).

8. Giulietti, A. (ed.) Laser-Driven Particle Acceleration Towards Radiobiology and Medicine (Springer, 2016).

9. Dover, N. P. et al. Effect of small focus on electron heating and proton acceleration in ultra-relativistic laser-solid interactions. Phys. Rev. Lett. 124, 1-6, DOI: 10.1103/PhysRevLett.124.084802 (2020).

10. Steinke, S. et al. Acceleration of high charge ion beams with achromatic divergence by petawatt laser pulses. Phys. Rev. Accel. Beams 23, 1-7, DOI: 10.1103/PhysRevAccelBeams.23.021302 (2020).

11. Sentoku, Y. et al. High-energy ion generation in interaction of short laser pulse with high-density plasma. Appl. Phys. B: Lasers Opt. 74, 207-215, DOI: 10.1007/s003400200796 (2002).

12. Kumar, S., Gopal, K. \& Gupta, D. N. Proton acceleration from overdense plasma target interacting with shaped laser pulses in the presence of preplasmas. Plasma Phys. Control. Fusion 61, DOI: 10.1088/1361-6587/ab216e (2019).

13. Ferri, J., Siminos, E. \& Fülöp, T. Enhanced target normal sheath acceleration using colliding laser pulses. Commun. Phys. 2, 1-8, DOI: 10.1038/s42005-019-0140-x (2019).

14. Gizzi, L. A. et al. Light Ion Accelerating Line (L3IA): Test experiment at ILIL-PW. Nucl. Instruments Methods Phys. Res. Sect. A Accel. Spectrometers, Detect. Assoc. Equip. 909, 160-163, DOI: 10.1016/j.nima.2018.03.016 (2018).

15. Vieira, J., Fiúza, F., Silva, L. O., Tzoufras, M. \& Mori, W. B. Onset of self-steepening of intense laser pulses in plasmas. New J. Phys. 12, DOI: 10.1088/1367-2630/12/4/045025 (2010).

16. Wilks, S. C., Kruer, W. L., Tabak, M. \& Langdon, A. B. Absorption of ultra-intense laser pulses. Phys. Rev. Lett. 69, 1383-1386, DOI: 10.1103/PhysRevLett.69.1383 (1992).

17. Tsung, F., Tochitsky, S. Y., Haberbergher, D. J., Mori, W. B. \& Joshi, C. Co2 laser acceleration of forward directed mev proton beams in a gas target at critical plasma density. J. Plasma Phys. 78, 373-382, DOI: 10.1017/S0022377812000189 (2012).

18. Chopineau, L. et al. Identification of Coupling Mechanisms between Ultraintense Laser Light and Dense Plasmas. Phys. Rev. X 9, 011050, DOI: 10.1103/PhysRevX.9.011050 (2019).

19. Nuter, R. et al. Influence of a preplasma on electron heating and proton acceleration in ultraintense laser-foil interaction. $J$. Appl. Phys. 104, DOI: 10.1063/1.3028274 (2008).

20. Babaei, J. et al. Rise time of proton cut-off energy in 2D and 3D PIC simulations. Phys. Plasmas 24, DOI: 10.1063/1. 4979901 (2017).

21. Gizzi, L. A. et al. A New Line for Laser-Driven Light Ions Acceleration and Related TNSA Studies. Appl. Sci. 7, 984, DOI: 10.3390/app7100984 (2017).

22. Strickland, D. \& Mourou, G. Compression of amplified chirped optical pulses. Opt. Commun. 56, 219-221, DOI: 10.1016/0030-4018(85)90120-8 (1985).

23. Gizzi, L. A. et al. Role of laser contrast and foil thickness in target normal sheath acceleration. Nucl. Instruments Methods Phys. Res. Sect. A Accel. Spectrometers, Detect. Assoc. Equip. 829, 144-148, DOI: 10.1016/j.nima.2016.01.036 (2016). 
24. Altana, C. et al. Investigation of ion acceleration mechanism through laser-matter interaction in femtosecond domain. Nucl. Instruments Methods Phys. Res. Sect. A-Accelerators Spectrometers Detect. Assoc. Equip. 829, 159-162, DOI: 10.1016/j.nima.2016.02.016 (2016).

25. Fonseca, R. A. et al. OSIRIS: A three-dimensional, fully relativistic particle in cell code for modeling plasma based accelerators. Lect. Notes Comput. Sci. (including Subser. Lect. Notes Artif. Intell. Lect. Notes Bioinformatics) 2331 LNCS, 342-351, DOI: 10.1007/3-540-47789-6_36 (2002).

26. Sgattoni, A., Londrillo, P., Macchi, A. \& Passoni, M. Laser ion acceleration using a solid target coupled with a low-density layer. Phys. Rev. E - Stat. Nonlinear, Soft Matter Phys. 85, 036405, DOI: 10.1103/PhysRevE.85.036405 (2012).

27. Pert, G. J. Two-dimensional hydrodynamic models of laser-produced plasmas. J. Plasma Phys. 41, 263-280 (1989).

\section{Acknowledgements}

This project has received funding from the CNR funded Italian research Network ELI-Italy and from the L3IA INFN Experiment of CSN5. We gratefully acknowledge support from CLF, STFC (UK) for in kind contribution to the experimental set up described in this experiment. E.B. acknowledges the OSIRIS Consortium, consisting of UCLA and IST, for the use of the OSIRIS 4.0 framework and the visXD framework. Simulations were performed on the supercomputers ARCHER (EPCC, UK) under Plasma HEC Consortium EPSRC grants EP/L000237/1 and EP/R029148/1 and Marconi-Broadwell (CINECA, Italy) under PRACE allocation.

\section{Author contributions statement}

L.A.G and L.L. conceived the experiment, L.A.G, L.L., F.Ba., F.Br., G.C., A.F., L.F., D.G., P.K. and D.P. conducted the experiment, L.A.G., L.L., F.B., F.B., G.C., A. F., D.G., P.K., D.P., and P.T. analysed the results. E.B. and P.J.B. carried out the numerical PIC simulations, L.L. carried out hydrodinamic simulations, L.A.G. and E.B. prepared the manuscript. All authors reviewed the manuscript. 
Figures

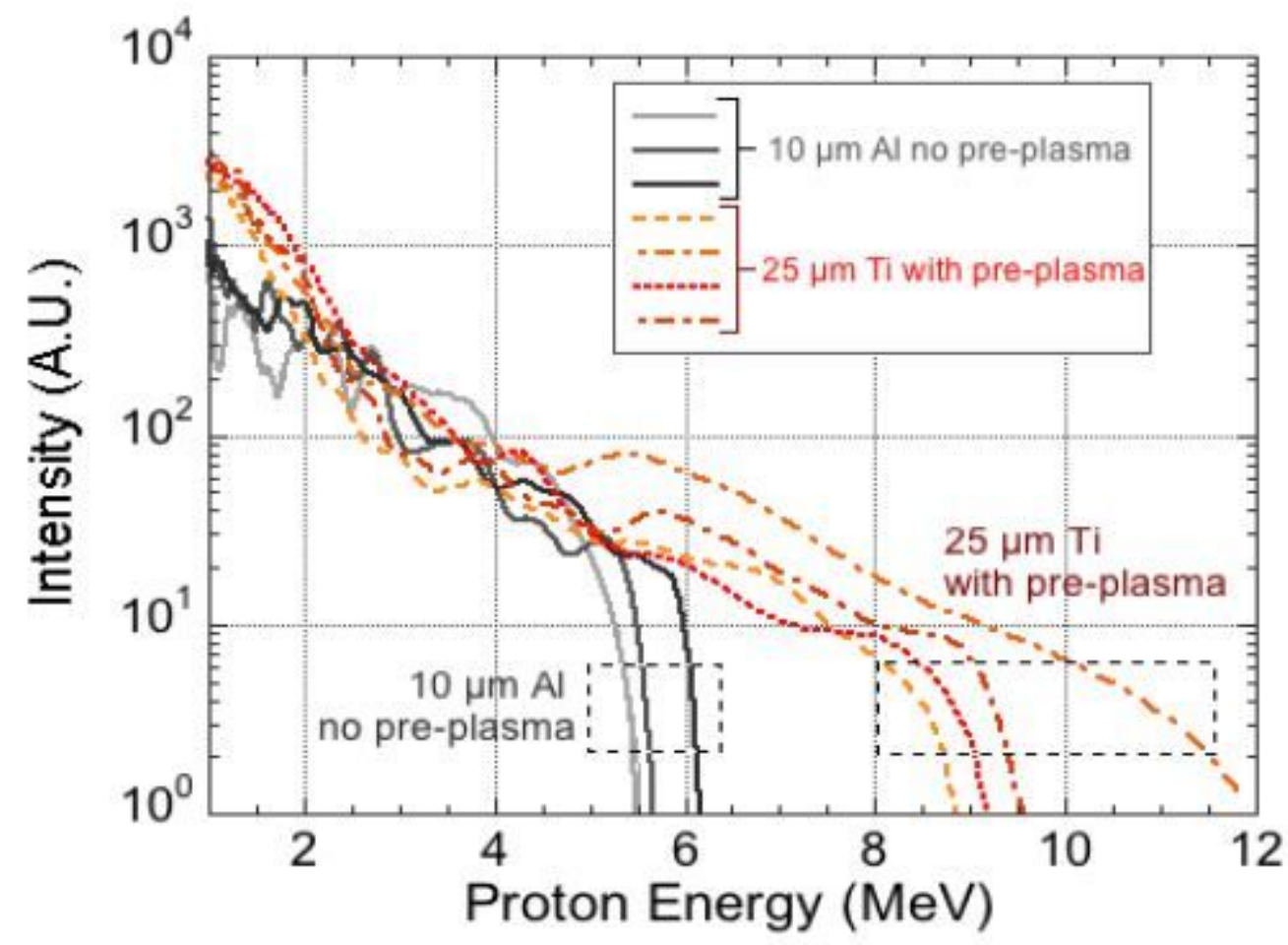

Figure 1

Comparison of experimental proton spectra obtained from the TPS for $10 \mu \mathrm{m}$ thick Al targets without preplasma and $25 \mu \mathrm{m}$ thick Ti target with the pre-plasma. 


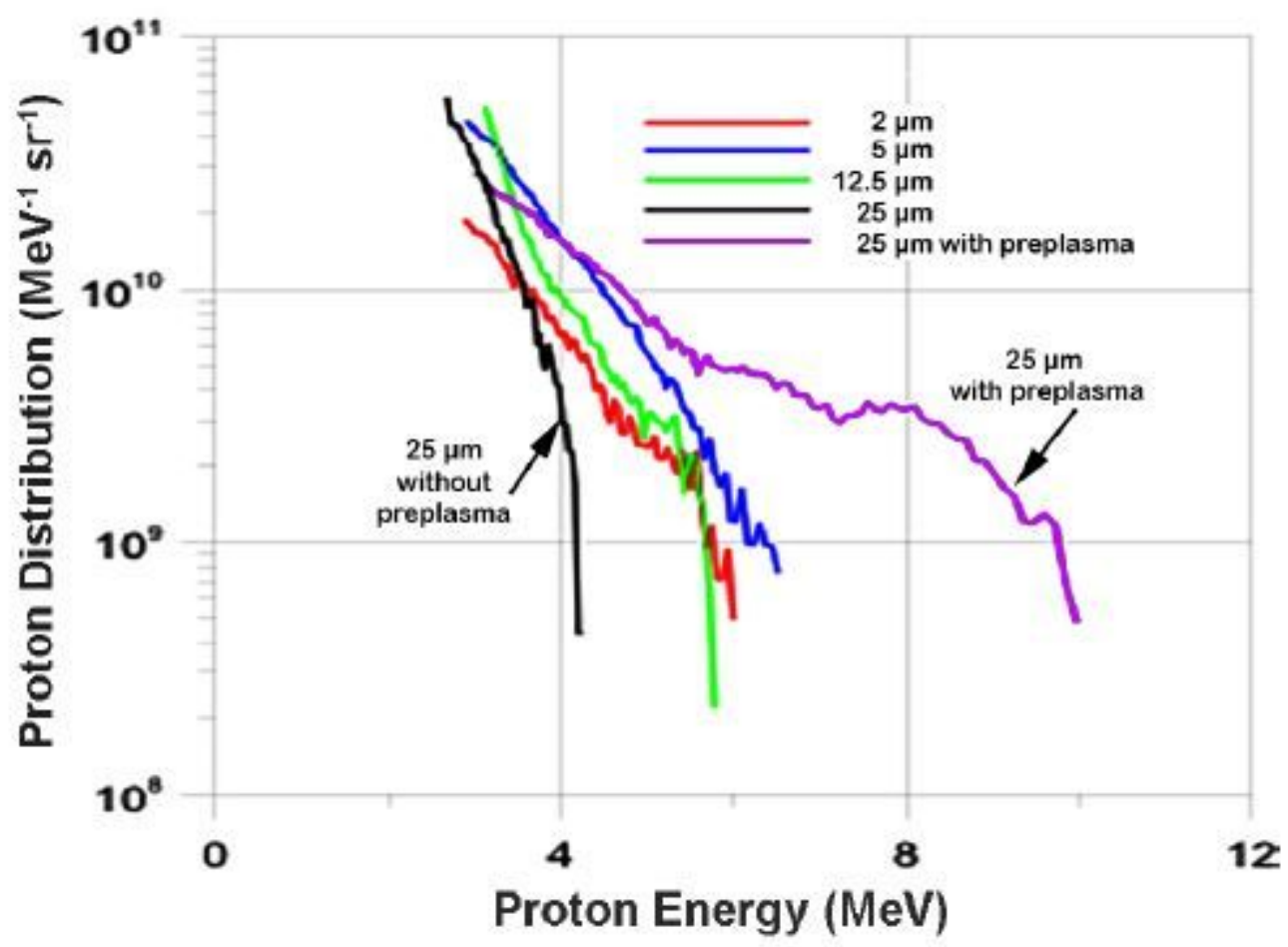

Figure 2

Comparison of experimental spectra obtained from deconvolution of the temporal evolution of the TOF signals from a set of Titanium foil targets of different thickness of 2, 5, 12.5 and $25 \mu \mathrm{m}$. All data were taken without the pre-plasma, except the purple line for $25 \mu \mathrm{m}$ with cut-off energy at $10 \mathrm{MeV}$.
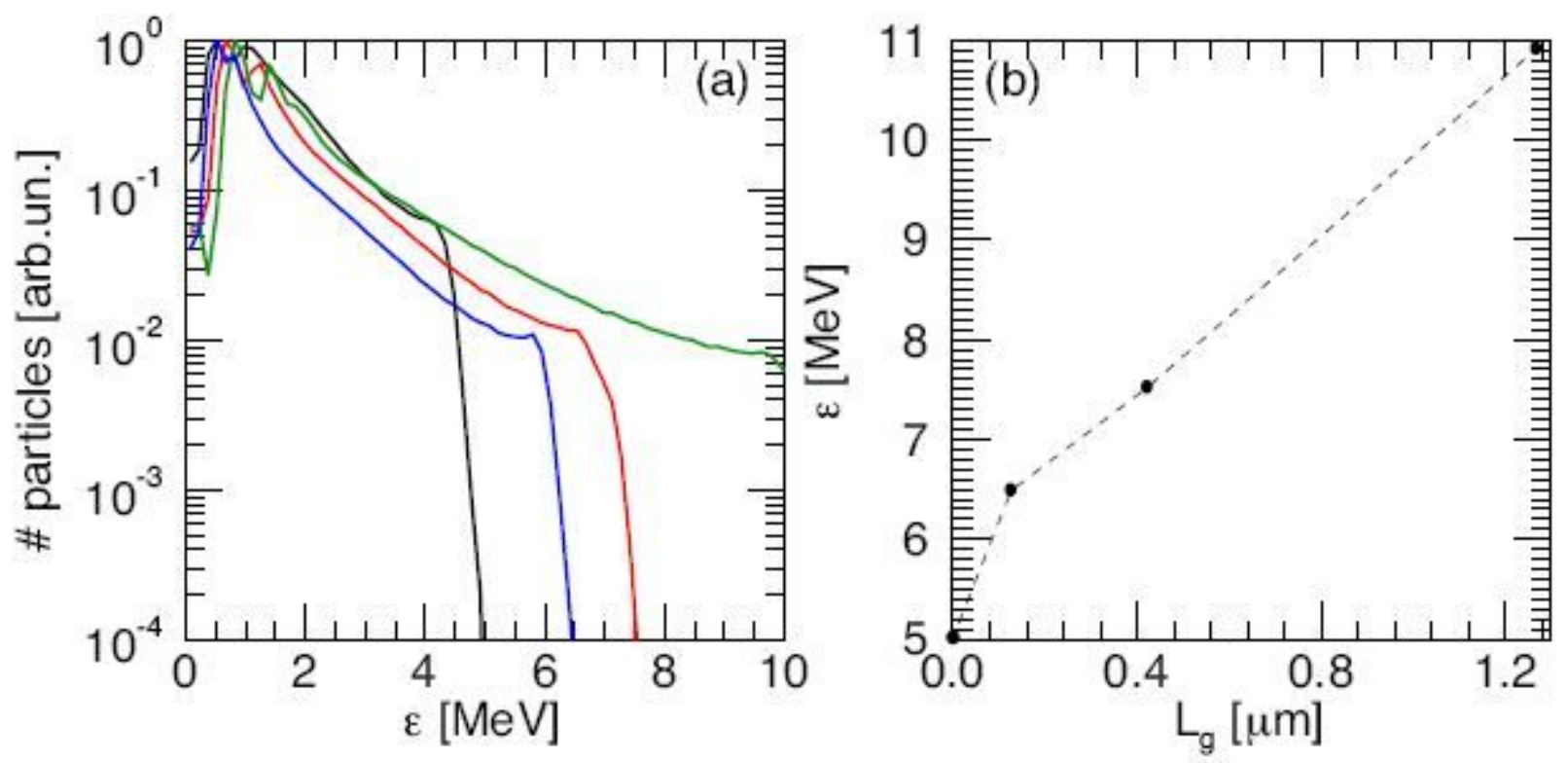

Figure 3 
3D PIC simulation results: (a) Proton energy spectra for a $10 \mu \mathrm{m}$ Al target for different pre-plasma scalength: $\mathrm{Lg}=0.13$ (blue), 0.42 (red) and $1.27 \mu \mathrm{m}$ (green). The black curve corresponds to a sharp plasma-vacuum transition. (b) Cut-off energy vs pre-plasma scalength for the cases in (a).

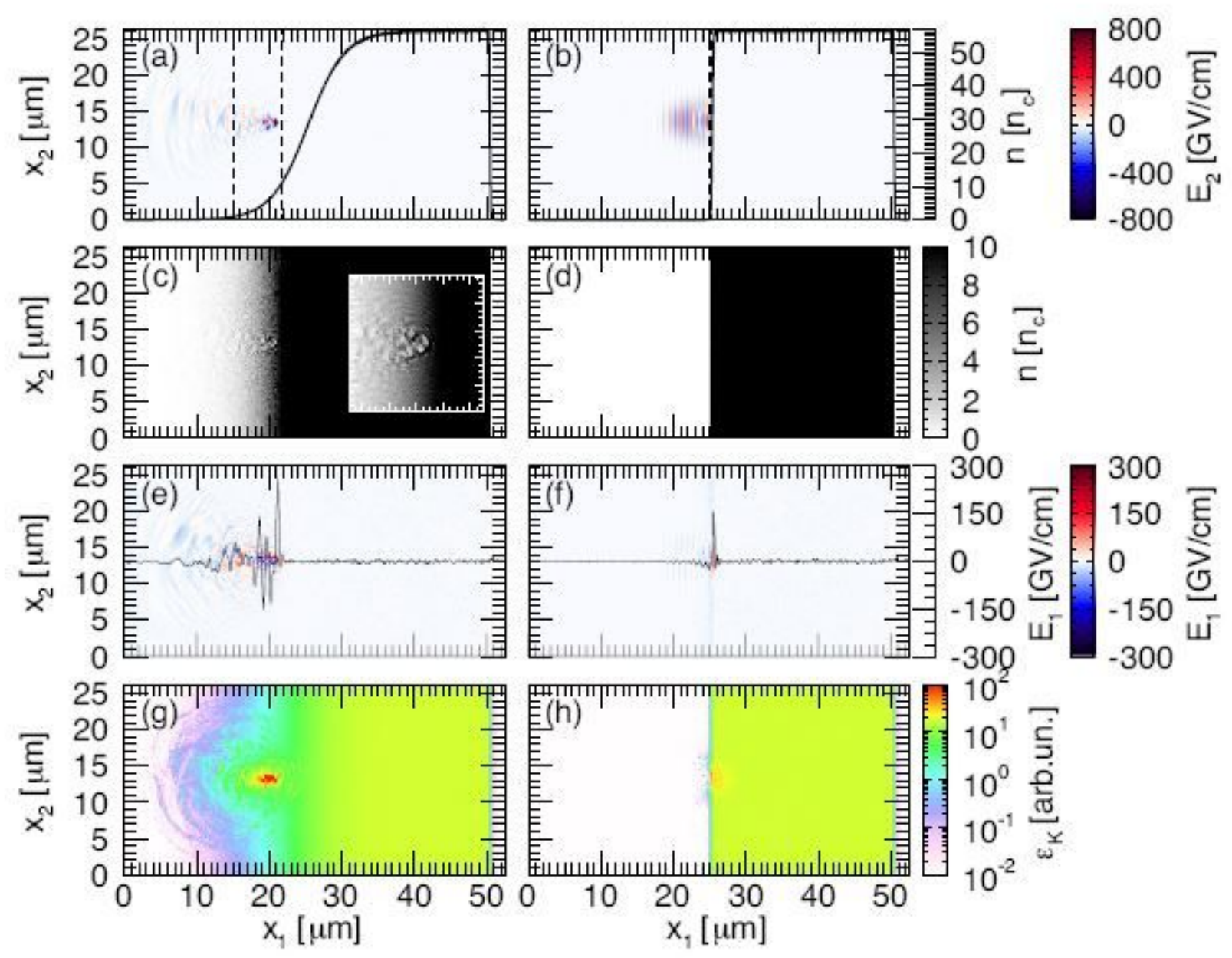

Figure 4

2D PIC simulations: ( $a, b)$ Laser electric field and longitudinal density profile (black solid line), (c, d) electron density, $(e, f)$ longitudinal electric field and $(g, h)$ electron kinetic energy density at $t=0.11$ ps for a Ti plasma with $\mathrm{Lg}=5$ (left column) and $0.13 \mu \mathrm{m}$ (right column). The black dashed lines in $(\mathrm{a}, \mathrm{b})$ denote the position of the critical density and the relativistic critical density. The inset in (c) is an enlargement showing details of the electron cavities on the target surface. The black solid lines in $(e, f)$ represent the electric field lineout in the middle of the simulation box. 

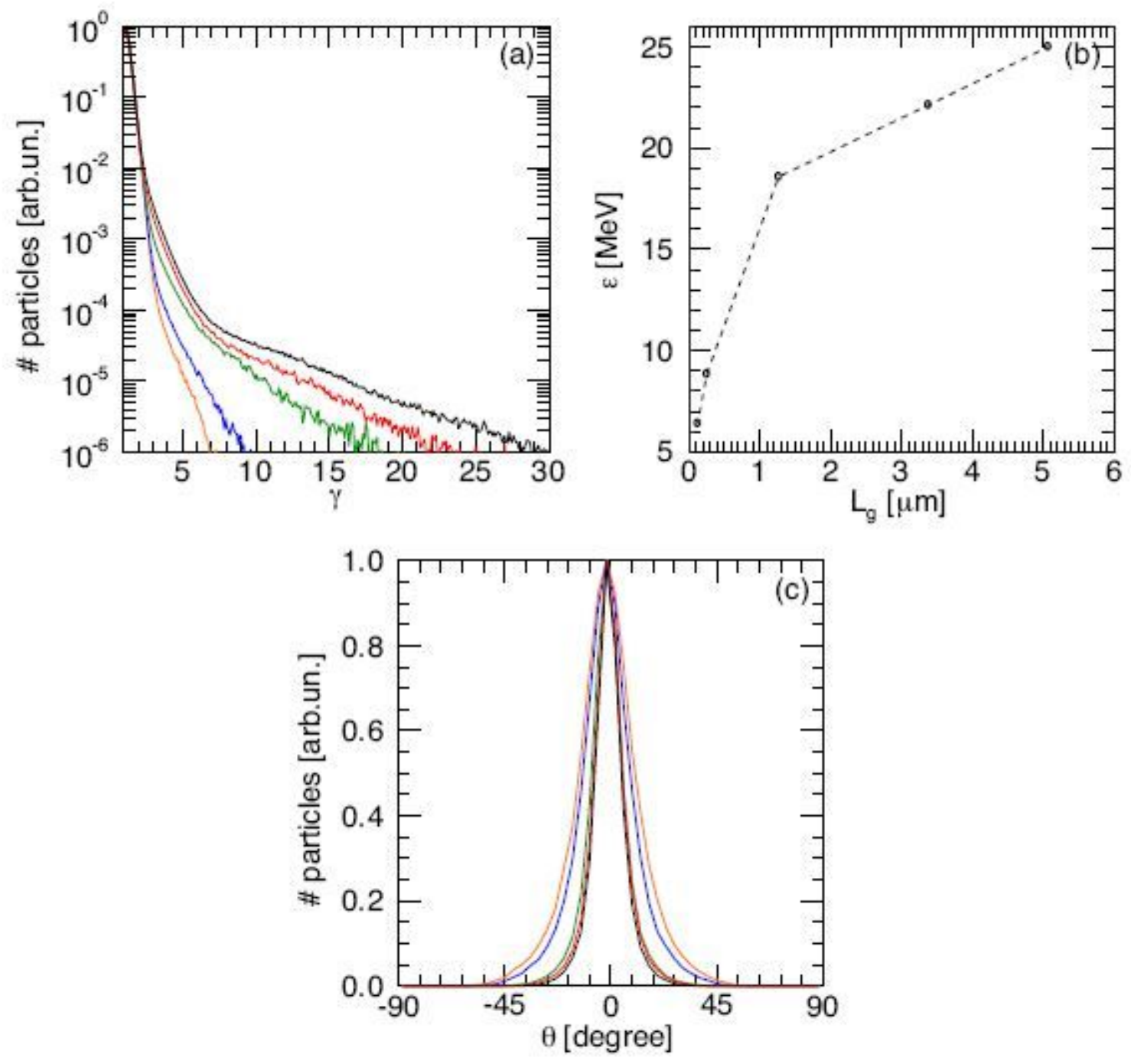

Figure 5

2D PIC simulations results: (a) Electron distribution for density scale-length $\mathrm{Lg}=0.13$ (orange), 0.25 (blue), 1.27 (green), 3.38 (red) and $5.07 \mu \mathrm{m}$ (black). (b) Proton cut-off energy vs pre-plasma scale length and (c) proton angular distribution for the cases in (a).

\section{Supplementary Files}

This is a list of supplementary files associated with this preprint. Click to download.

- GizzietallnfluenceOfpreplasmaSuppIMat.pdf 